\title{
Letter to the Editor: Limitations to the Measurement of Oxygen Concentrations by HRTEM Imposed by Surface Roughness
}

In an article published in Microscopy and Microanalysis recently (Jia et al., 2004), it was claimed that aberrationcorrected high resolution transmission electron microscopy (HRTEM) allows the quantitative measurement of oxygen concentrations in ceramic materials with atomic resolution. Similar claims have recently appeared elsewhere, based on images obtained through aberration correction (Jia et al., 2003; Jia \& Urban, 2004) or very high voltages (Zhang et al., 2003). Seeing oxygen columns is a significant achievement of great importance (Spence, 2003) that will doubtlessly allow some exciting new science; however, other models could provide a better explanation for some of the experimental data than variations in the oxygen concentration. Quantification of the oxygen concentrations was attempted by comparing experimental images with simulations in which the fractional occupancy in individual oxygen columns was reduced. The results were interpreted as representing nonstoichiometry within the bulk and at grain boundaries. This is plausible because previous studies have shown that grain boundaries can be nonstoichiometric (Kim et al., 2001), and it is indeed possible that oxygen vacancies are present at boundaries or in the bulk. However, is this the only possible interpretation? We show that for the thicknesses considered a better match to the images is obtained using a simple model of surface damage in which atoms are removed from the surface, which would usually be interpreted as surface damage or local thickness variation (from ion milling, for example).

This alternative interpretation is suggested by the observation that there are also changes to the other column intensities, which are at least as significant as the changes seen at the O columns, for example, Figure 7 of Jia et al. (2004) or Figure 1 of Jia and Urban (2004). As a specific example, consider Figures 1e and 2 of Jia et al. (2003). Those simulations reveal that the intensity measured at an oxygendeficient oxygen column does indeed decrease, in agreement with the experimental images. However, the simulations also show that the neighboring Ti columns appear slightly brighter than in the bulk, an observation not supported by the experimental data. In fact, in some places the nearby $\mathrm{Ti}$ columns appear significantly dimmer. This is shown most clearly in Figure 2a of Jia et al. (2003).
We show that removing atoms from the surface explains both observations by a single mechanism. Figure 1a of this letter shows a simulation of oxygen vacancies, modeled via fractional occupancy, with parameters matching those of Jia et al. (2003), which agrees well with the simulation given in Figure $2 \mathrm{c}$ of that paper. Figure 1 also shows simulations for surface roughness modeled by a partial surface step (b), and a full surface step (c), as defined in (d) and the figure caption. From this figure it is apparent that reduced occupancies and surface roughness both introduce changes in the intensity of the oxygen columns. However, only the latter phenomenon introduces notable decreases in the intensities of the neighboring Ti columns. The nearby $\mathrm{Ti}$ columns appear slightly brighter near an $\mathrm{O}$ column with reduced occupancy and generally appear dimmer in the presence of surface damage. This is only intended to be a simple model for the effect of surface roughness to use in the simulation, and other models might be more appropriate, such as surface relaxations, adsorbates, or amorphous layers, which might be several tenths of a nanometer thick.

Nevertheless, this simple surface roughness model clearly compares well with the experimental data in Figure 2a of Jia et al. (2003), suggesting that the observed intensity changes are better fitted by some form of surface roughness rather than oxygen vacancies in the bulk. There are many other possible models of surface damage effects from ion beam thinning or other sample preparation techniques. Preferential thinning at boundaries could, in some circumstances, concentrate these effects at grain boundaries. We would expect the presence of a thin surface layer on HRTEM specimens that would be reconstructed, amorphous, or damaged. There is also the possibility of electron beam damage or surface contamination that varies over the sample. These effects could be more random than the sharp step in our simulations, allowing a closer match to the observed intensity variation. Even if some technique were used to anneal the surface, we would still expect surface steps to be present similar to those we model.

Finally, Jia et al. (2004) echo the sentiment, stated explicitly by Jia and Urban (2004), that Z-contrast imaging in scanning transmission electron microscopy (STEM) "does 
(a)

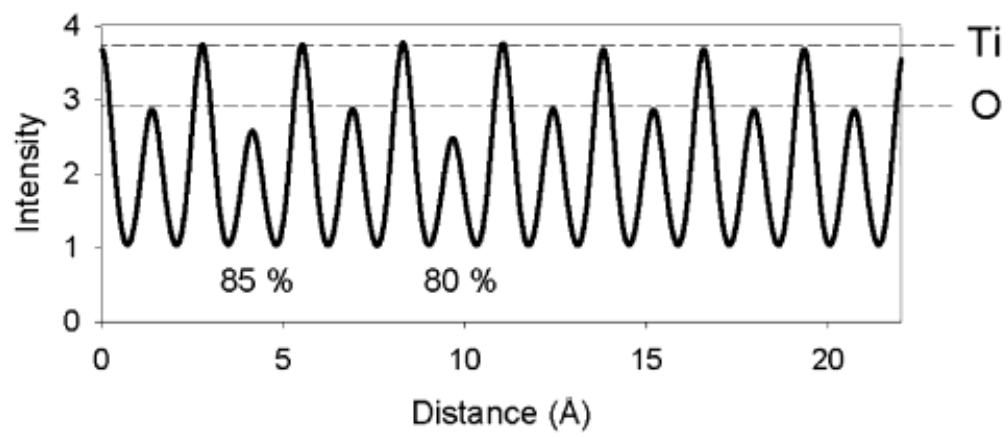

(b)

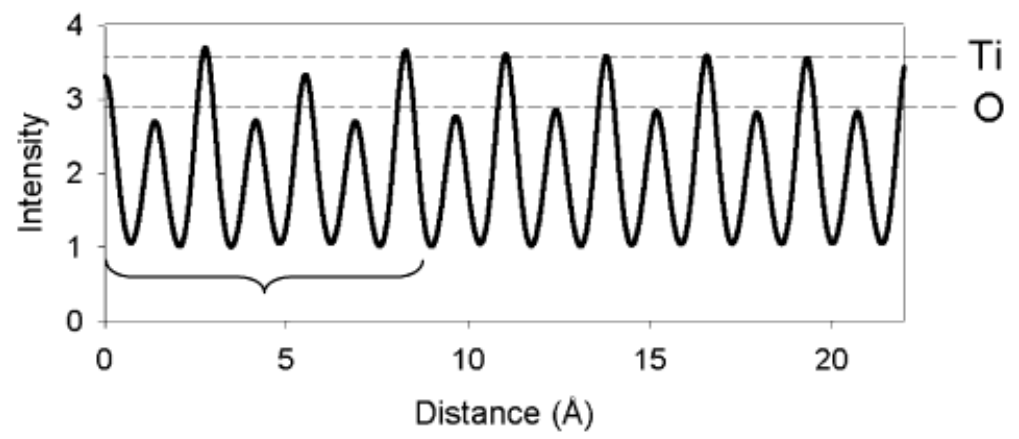

(c)

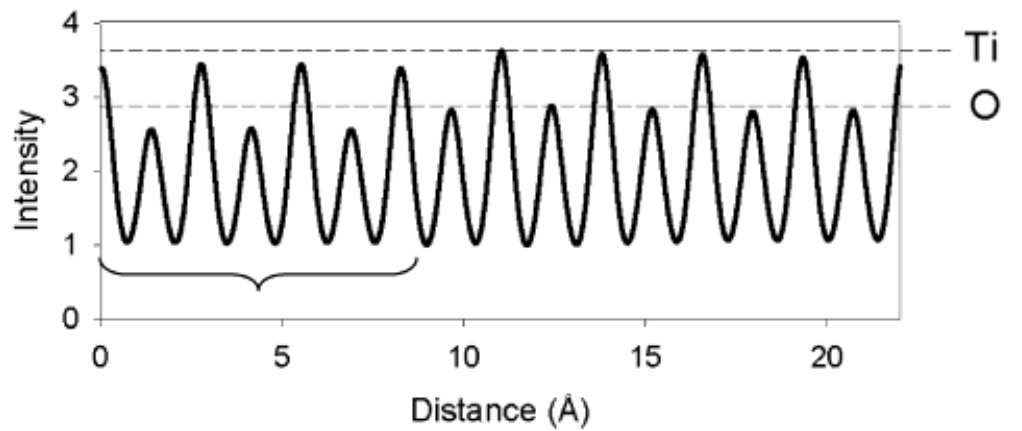

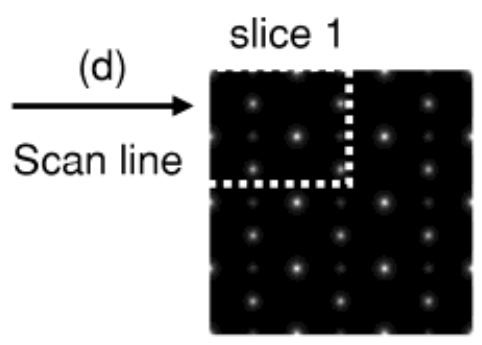

slice 2

slice 3
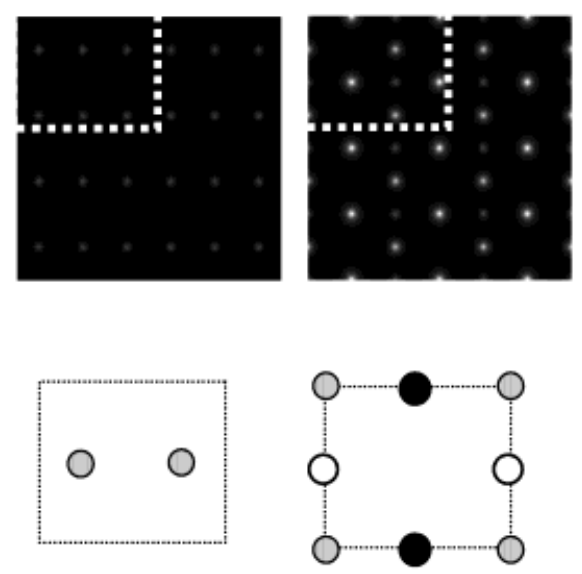

slice 4

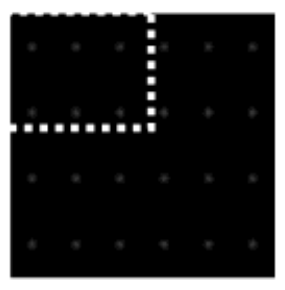

(e)
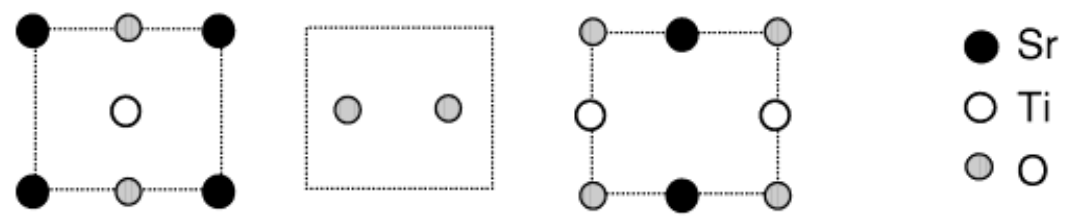

Figure 1. Linetrace simulations of the (HRTEM) image intensity for $\mathrm{SrTiO}_{3}$ [011] for the conditions given by Jia et al. (2003) $\left(E=200 \mathrm{kV}, C_{s}=-0.04 \mathrm{~mm}, Z=+8 \mathrm{~nm}\right.$, aperture $=20 \mathrm{mrad}, 4 \mathrm{~nm}$ thick, information limit $\left.7.7 \mathrm{~nm}^{-1}\right)$ for (a) fractional occupancy in two O columns as labeled, (b) partial surface step located above the brace, and (c) full surface step located above the brace. In all cases, the taller peaks represent the Ti columns and the smaller peaks are the $\mathrm{O}$ columns. The intensity values in stoichiometric $\mathrm{SrTiO}_{3}$ are shown with dotted lines as a visual aid. d: Projected potentials of the four even slices of the repeat distance. In a partial surface step, we omit the marked areas in slices 3 and 4 of the final repeat unit; in a full step, we omit the marked areas in all slices of the final repeat unit. The arrow points to the line from which the extracted scans are taken. e: Atom locations in the repeating unit are shown for slices 1-3 (slice 4 is identical in structure to slice 2). 
not allow oxygen to be imaged because it is confined to elements with a high value of the nuclear charge Z." This is a misconception because, although the oxygen columns could not be resolved in earlier work (Kim et al., 2001), there is no fundamental reason why this should always be so. In fact recent work has since shown that, following aberration correction, oxygen columns can be resolved in Z-contrast STEM, even in the presence of heavier columns (Chisholm et al., 2004). Further calculations (Cosgriff et al., 2005) suggest that column-by-column electron energy loss spectroscopy may allow quantitative analysis of a single column in a manner that is not so strongly dependent on the atomic number or surface damage.

In summary, we have shown that effects other than oxygen vacancies better account for contrast in recent experimental images. Unless these alternative explanations can be definitively excluded, there would seem to be no justification for making assertions about the oxygen concentration in the bulk. Given these results, we suggest that "seeing is believing" (Spence, 2003) is not necessarily true in this context.

Andrew R. Lupini, Matthew F. Chisholm, and Klaus van Benthem

Condensed Matter Sciences Division

Oak Ridge National Laboratory

Oak Ridge, TN 37831, USA

Leslie J. Allen, Mark P. Oxley, and Scott D. Findlay

School of Physics, University of Melbourne

Victoria 3010, Australia
Maria Varela and Stephen J. Pennycook Condensed Matter Sciences Division Oak Ridge National Laboratory

Oak Ridge, TN 37831, USA

\section{RefERENCES}

Chisholm, M.F., Lupini, A.R., Pennycook, S.J., Ohkubo, I., Christen, H.M., Findlay, S.D., Oxley, M.P. \& Allen, L.J. (2004). Simultaneous $z$-contrast and phase contrast imaging of oxygen in ceramic interfaces. Microsc Microanal 10(Suppl 2), 256-257.

Cosgriff, E.C., Oxley, M.P., Allen, L.J. \& Pennycook, S.J. (2005). The spatial resolution of imaging using core-loss spectroscopy in the scanning transmission electron microscope. Ultramicroscopy 102, 317-326.

Jia, C.L., Lentzen, M. \& Urban, K. (2003). Atomic-resolution imaging of oxygen in perovskite ceramics. Science 299, 870-873.

Jia, C.L., Lentzen, M. \& Urban, K. (2004). High-resolution transmission electron microscopy using negative spherical aberration. Microsc Microanal 10, 174-184.

Jia, C.L. \& Urban, K. (2004). Atomic-resolution measurement of oxygen concentration in oxide materials. Science 303, 2001-2004.

Kim, M., Duscher, G., Browning, N.D., Sohlberg, K., PanTelides, S.T. \& PENNYCOoK, S.J. (2001). Non-stoichiometry and the electrical activity of grain boundaries in $\mathrm{SrTiO}_{3}$. Phys Rev Lett 86, 4056-4059.

Spence, J.C.H. (2003). Oxygen in crystals—Seeing is believing. Science 299, 839-841.

Zhang, Z., Sigle, W., Phillipp, F. \& Ruhle, M. (2003). Direct atom-resolved imaging of oxides and their grain boundaries. Science 302, 846-849.

\section{Authors' Response}

The authors are grateful to the Editor for the opportunity to reply to the letter by Lupini et al. The authors of the above letter comment on a set of recent articles in which the novel technique of imaging at a negative value of the spherical aberration coefficient of the objective lens in an aberrationcorrected transmission electron microscope (NCSI technique) is methodically described and applied to the measurement of the occupancy of atomically resolved oxygen columns in perovskites. In particular, the authors raise doubts about the possibility of inferring quantitative data from measurements of the local image intensity at the position of the oxygen atom columns. With reference to the study by Jia et al. (2003a), the letter authors present an image simulation on the basis of which it is stated that the observed effect of a reduced intensity at the oxygen atomic columns should not be interpreted in terms of reduced oxygen occupancy but can, as the authors claim, be "better" explained on the basis of the effect of surface roughness on contrast. In addition, the authors emphasize the work of Kim et al. (2001) with respect to the nonstoichiometry of the oxygen occupancy in grain boundaries of $\mathrm{SrTiO}_{3}$ and criticize our reference to the literature in which it is reported that oxygen cannot be observed by the scanning transmission electron microscopy (STEM) technique in $\mathrm{Z}$-contrast. In the following, we shall demonstrate that in spite of the fact that a nonideal surface morphology can-as in the application of any (!) electron microscopic technique whether used in TEM or in STEM-have an effect on local 
image intensity, meaningful quantitative measurements of relative oxygen-atom site occupancies can be carried out employing the NCSI technique.

The NCSI technique applied in the aberration-corrected transmission electron microscope provides transmission electron microscopy with a technique allowing atomic-resolution studies on oxygen and other low-nuclear-charge atomic species at enhanced bright-atom contrast at a minimum of contrast delocalization. This technique was first described by Jia et al. (2003a). In that study we also briefly mentioned the possibility of employing the NCSI technique to quantitative studies on the oxygen occupancy of atomic columns in the center and the vicinity of defect structures. In Figure 2 of that article a first example was given in the form of a "point defect" showing reduced intensity at an oxygen position. No attempt was made in that paper to present any detailed study because this would have been beyond the scope of the publication, whose purpose was to introduce the new imaging technique. We also did not interpret the derived occupancy in terms of bulk concentrations of oxygen because, and this is well known, the contrast does not allow us to differentiate between surface and volume defects. Truly, such an interpretation would require additional information.

Lupini et al. refer to Figure 2 of Jia et al. (2003a), claiming that the intensities of two Ti columns neighboring an oxygen column in $\mathrm{SrTiO}_{3}$ are dimmer if the respective oxygen column shows reduced intensity. This conclusion is not justified. By visual inspection of Figure 2 it is impossible to make any statement on such differences because they are simply not visible in the print of the electron microscopic image. The only relevant basis for a quantitative interpretation of the image contrast is the intensity trace taken from the original data (Fig. 2A). In that figure, which is reproduced as Figure 1 of this comment, the intensity peak (a) at the Ti position left of defect " 1 " is slightly lower than the neighboring peak further left. However, the reduction is in the same order of magnitude as the average variation of the Ti peak height (see the Ti peaks on the right of Fig. 1). The peak (b) to the right of " 1 " is significantly reduced in intensity. At defect "2" the Ti peak (c) to the left shows essentially the same intensity as the other "normal" $\mathrm{Ti}$ peaks, whereas peak (d) to the right shows increased intensity. Because Lupini et al. have no other basis for their statements, the conclusion that the Ti atom intensity in our images is-as a general phenomenon-reduced, is wrong. This also forbids us from concluding, on the basis of their image simulations, that surface irregularities are a compelling origin of the reduction of oxygen-column intensity. In this respect we add two comments: First, the authors are citing two more figures, Figure 7 of Jia et al. (2004a) and Figure 1 of Jia and Urban (2004), which they claim would support their statement that the Ti atom column intensity is reduced at defect-containing oxygen atom columns. Both interpretations are wrong and misleading. In the former, an intensity trace is given depicting only the oxygen atom

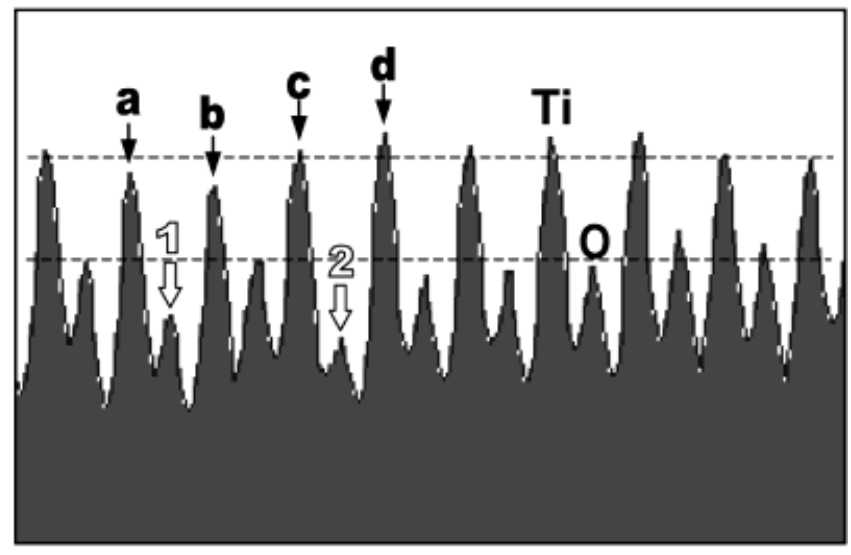

Figure 1. Intensity profile of $\mathrm{Ti}$ and $\mathrm{O}$ columns of Jia et al. (2003a) reproduced in Figure 2a. The horizontal lines show the mean values of the intensity peaks. The deviation of the individual peaks from the mean value demonstrates the data scatter that is not avoidable in real experiments. The reduction of the intensity of the oxygen peaks 1 and 2 is evidently far beyond the range of data scatter. The estimation of the occupancy of the oxygen columns 1 and 2 is based on the mean value instead of a specific peak height. It is clear that the reduction of the intensity of the $\mathrm{Ti}$ columns $\mathrm{a}$ and $\mathrm{b}$ is within the range of the scattering of the data. Furthermore the intensity trace (taken from the original and unprocessed CCD camera data) shows not only reduced but also equal and higher intensity at the $\mathrm{Ti}$ atom positions neighboring oxygen columns of reduced intensity.

intensity and no Ti atom intensity at all. In the latter, no intensity traces are given, and the image prints are clearly excluding any quantitative statement with respect to $\mathrm{Ti}$ atom intensity. Second, our own image simulations on the effect of Ti atom nonstoichiometry on the intensity yield a much stronger effect on intensity than that described by Lupin et al., if one of the Ti atoms in a Ti atom column is replaced by a vacancy. In addition, the effect depends on imaging parameters and sample thickness. In conclusion, we find that the claim by Lupini et al. that there is compelling evidence that our observations are to be attributed to surface damage is unfounded.

A detailed study on oxygen occupancy measurements was carried out by Jia and Urban (2004). The example chosen was the $\Sigma 3\{111\}$ twin boundary in $\mathrm{BaTiO}_{3}$ thin films. In this study care was taken to exclude, as far as this was feasible, any effect of surface irregularities by deriving from the intensity data a relative occupancy of oxygen atom columns, that is, the difference in the column occupancy in the defect with respect to the column occupancy in an area far away from the defect. Because this can only eliminate effects that are common to areas close and far from the defect, we employed additional measurements that should indicate particular surface and other irregularities pertaining only to the defect area itself. The possibility of preferential surface damage or preferential thinning by means of 
ion-beam preparation, mentioned by Lupini et al., belong to this category. To obtain an indication of the possibility that such effects could affect our results, we measured also the $\mathrm{BaO}$ intensity in the boundary as well as the $\mathrm{Ti}$ and $\mathrm{O}$ signals on both sides of the boundary plane. This is explicitly noted by Jia and Urban (2004) in the text. The data are not given in Figure 4 of that article but were presented at two recent conferences (Jia et al. 2003b, 2004b). For all the measured intensities, the intensities do not deviate from the "bulk" values measured at a larger distance from the defects. This is strong evidence for the fact that the surface morphology of our samples is not substantially, if at all, modified by the presence of the twin boundary on which the oxygen occupancy measurements were carried out. We therefore reject the criticism by Lupini et al. that our results are unreliable because surface effects were allegedly not taken into account. Unfortunately, Lupini et al. imply, for the uninitiated reader, that these problems are not discussed in Jia and Urban (2004). On the contrary, we have carried out extensive image simulation studies on the impact of surface irregularities on our results, and we conclude in the discussion part of our article that "atomic scale surface roughness" cannot be excluded and could account for the observed scatter in our data, that is for the determined standard deviation.

Because our measurement on the oxygen occupancy in the $\Sigma 3\{111\}$ boundary is the first of its kind, we have looked for independent support for our results. This is found in a study by Grey et al. (1998) in which, by chemical means, oxygen-vacancy concentrations in the hexagonal phase of $\mathrm{BaTiO}_{3}$ are studied by doping with $\mathrm{Fe}^{3+}$ on $\mathrm{Ti}^{4+}$ sites. As described by Jia and Urban (2004), their results agree well with our measurements, which corroborates our conclusion that the measured data refer to the volume oxygen occupancy.

Finally, we comment on our reference to Kim et al. (2001). First of all, the conclusion in this article on an oxygen deficiency of a grain boundary in $\mathrm{SrTiO}_{3}$ is arrived at after an involved procedure in which the EELS spectra on $8^{\circ}[001]$ tilt grain boundaries are combined with Z-contrast results on $36^{\circ}$ [001] boundaries. Because the EELS results cannot be interpreted directly, theoretical electron structure calculations are carried out on $53^{\circ}\{210\}$ tilt grain boundaries that are, most regrettably, not those investigated experimentally. Their article not only illustrates that the STEM technique is quite involved, but that the results are subject to discussion. It also demonstrates how urgent it is to investigate the important question of oxygen deficiency of lattice defects in perovskites by alternative techniques. We also have to mention that in none of the recent publications on the application of the STEM technique are the effects of surface irregularities on the observations discussed, in spite of the fact that this technique is not free of the possibility of such artifacts.

As Lupini et al. admit, Kim et al. (2001) explicitly state that oxygen is not accessible by the Z-contrast STEM tech- nique. Also, a detailed literature search did not reveal any evidence of atomic oxygen imaging by the STEM technique until the submission and publication of the article by Jia and Urban (2004). It is surprising that Lupini et al. characterize our correct references to their own publications as our personal "sentiment" and, against all accepted rules, are themselves referring to unpublished results.

In conclusion, we have provided detailed evidence that the attempt by Lupini et al. to bring discredit upon our published results concerning oxygen occupancy measurements employing the NCSI technique in the aberrationcorrected transmission electron microscope is not justified. The problem of the effect of surface irregularities on contrast is well known in high-resolution electron microscopy. None of the present electron microscopy techniques, whether applied in TEM or STEM, is a priori free from this inevitable shortcoming. The only solution is to obtain additional evidence allowing us to draw conclusions about the particular observation conditions. This has been done, to the best of our current knowledge, in the work by Jia and Urban (2004) with the result that, as far as this can be judged, confidence is justified in oxygen occupancy measurements, which have a bearing on the science of grain boundary nonstoichiometry in perovskites.

Chun-Lin Jia, Markus Lentzen, and Knut Urban Institute for Solid State Research and

Ernst Ruska Center for Microscopy and Spectroscopy with Electrons, Research Center Juelich D-52425 Juelich, Germany

\section{REFERENCES}

Grey, I.E., Li, Ch., Cranswick, L.M.D., Roth, R.S. \& Vanderah, T.A. (1998). Structure analysis of the $6 \mathrm{H}-\mathrm{Ba}\left(\mathrm{Ti}, \mathrm{Fe}^{3+}, \mathrm{Fe}^{4+}\right) \mathrm{O}_{3-\delta}$ solid solution. J Solid State Chem 135, 312-321.

Jia, C.L., Lentzen, M. \& Urban, K. (2003a). Atomic-resolution imaging of oxygen in perovskite ceramics. Science 299, 870-873.

Jia, C.L., Lentzen, M. \& Urban, K. (2003b). Quantitative aberration-corrected transmission electron microscopy. In FEMMS 2003, San Francisco, CA.

Jia, C.L., Lentzen, M. \& Urban, K. (2004a). High-resolution transmission electron microscopy using negative spherical aberration. Microsc Microanal 10, 174-184.

Jia, C.L., Lentzen, M. \& Urban, K. (2004b). Quantitative aberration-corrected transmission electron microscopy. In $M \nLeftarrow M$ Pre-Meeting on Electron Microscopy in an Aberration-Corrected Environment, Savannah, GA.

Jia, C.L. \& Urban, K. (2004). Atomic-resolution measurement of oxygen concentration in oxide materials. Science 303, 2001-2004.

Kim, M., Duscher, G., Browning, N.D., Sohlberg, K., PanTelides, S.T. \& Pennycook, S.J. (2001). Nonstoichiometry and the electrical activity of grain boundaries in $\mathrm{SrTiO}_{3}$. Phys Rev Lett 86, 4056-4059. 